Kompass

Dermatologie

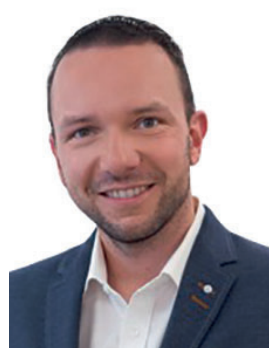

Mario Mader

Koordination «DermaCampus»

\title{
DermaCampus in den Zeiten von COVID-19
}

\begin{abstract}
Vieles hat sich in den vergangenen Wochen geändert. Als der Plan für den Inhalt dieser Ausgabe des DermaCampus gemacht wurde, war noch nicht abzusehen, wie stark der Coronavirus unseren Alltag beeinflussen wird. Nun steht uns ein «gewaltiger Wandel» bevor, aber nicht ganz so, wie ihn Professor Dr. Peter Rohmeiss in unserem Beitrag «Arztberuf im Wandel - welche Herausforderungen erwarten uns?» auf uns zukommen sieht. Dennoch können wir auch ein bisschen Beständigkeit im neuesten DermaCampus bieten. Mit seinem Beitrag zur operativen Lasermedizin setzt Prof. Dr. Stefan Hammes die Reihe rund um die Anwendung von Lasern in der Dermatologie fort und bringt uns auf den aktuellen Stand. Und übrigens, wenn Sie auf der Suche nach aktuellen Informationen aus der Forschung rund um COVID-19 sind, lohnt sich ein Blick auf die Homepage des Karger Verlags. Dort sind nun relevante wissenschaftliche Artikel frei zugänglich.
\end{abstract}

\section{Frei zugängliche Informationen zu COVID-19}

Auf der Homepage des Karger Verlags finden Sie hilfreiche Informationen zu COVID-19:

- freier Zugang zu relevanten wissenschaftlichen Artikeln aus dem Karger-Programm

- interaktives 3D-Media-Asset

www.karger.com/Company/covid19

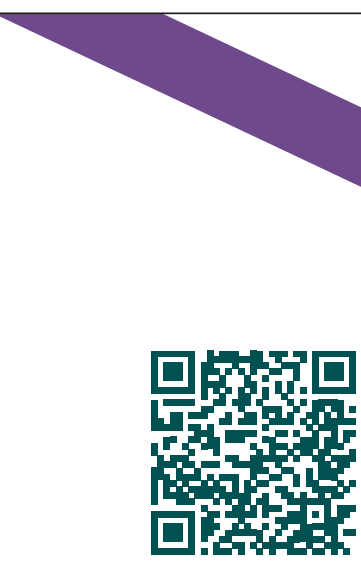

3D-Media-Asset

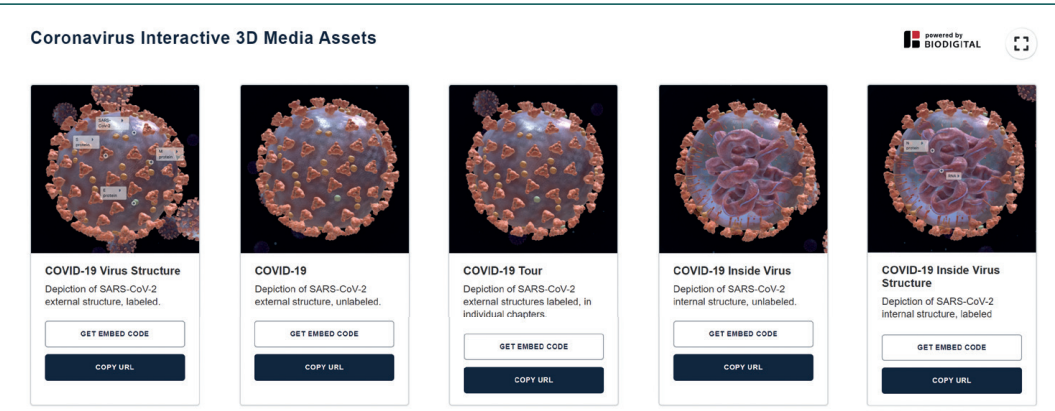

Karger's 


\section{Aus der Praxis \\ Operative Lasermedizin}

Die Einsatzgebiete von Lasern sind sehr vielseitig. Für welchen Zweck ein Lasergerät eingesetzt werden kann, hängt entscheidend vom Lasermedium und der Betriebsart ab. Das Lasermedium bestimmt die Wellenlänge und damit nach der Theorie der selektiven Photothermolyse das Zielchromophor. Die Betriebsart und hier vor allem die Impulsdauer bestimmt zusätzlich die biophysikalischen Eigenschaften der abgegebenen photonischen Energie.

In der ästhetischen Dermatologie werden häufig kurze Impulse im Milli-/Mikro-/Nano- und Pikosekundenbereich verwendet, um Zielstrukturen zu zerstören, ohne das umgebende Gewebe zu beeinflussen.

Im Bereich der operativen Dermatologie, aber auch in vielen anderen operativen Fächern, werden Laser oft als Alternative zum Skalpell eingesetzt. Hier will man vor allem die schneidende, abtragende oder koagulierende Wirkung nutzen, oft in Verbindung mit der blutstillenden Eigenschaft mancher Lasersysteme.

Das wichtigste Zielchromophor in der operativen Lasermedizin ist Wasser. Folglich benötigt man Laser mit Wellenlängen, die gut in Wasser absorbiert werden und gegebenenfalls eine große Eindringtiefe haben. Vorrangig kommen daher folgende Infrarot-Lasersysteme zum Einsatz.

\section{Erbium: YAG-Laser (2940 nm)}

Die Wellenlänge dieses Lasers hat den größten Absorptionskoeffizienten in Wasser. Daher wird die Laserenergie bereits in den oberen Schichten der Epidermis/Dermis durch das intrazelluläre Wasser absorbiert und es erfolgt eine explosionsartige Ablation ohne Freisetzung nennenswerter Wärmemengen (kalte Ablation). Darum hat der Erbium:YAG-Laser auch keine hämostatische Wirkung, er trägt sehr schonend Gewebe in 30-80 um dicken Schichten ab, ohne die darunter liegenden Gewebeschichten wesentlich zu beeinflussen. Indikationen in der Dermatologie sind die Ablation benigner Tumore, Skinresurfacing, Blepharoplastiken, Laser assisted drug delivery und andere. Obligat sollte eine Rauchabsaugung eingesetzt werden, da die vaporisierten Gewebepartikel bei entsprechender Patientenanamnese gegebenenfalls nicht inaktivierte Viruspartikel enthalten können. In diesen Fällen sollte die Indikationsstellung gründlich überdacht und möglicherweise andere Therapieoptionen bevorzugt werden.

\section{$\mathrm{CO}_{2}$-Laser (10600 $\mathrm{nm}$ )}

Die Wellenlänge dieses Lasers hat immer noch einen sehr großen Absorptionskoeffizienten in Wasser, jedoch ist der Koeffizient fast eine Größenordnung kleiner als der des Erbium:YAG-Lasers. Dies führt ebenso zu einer oberflächlichen Ablation, jedoch entsteht hier aufggrund der nicht ganz so explosiven Abtragung eine grö- ßere Wärmekomponente. Diese führt einerseits zur Blutstillung, beeinflusst andererseits aber auch tiefere Gewebeschichten, hier vor allem das Kollagen der Dermis. Indikationen in der Dermatologie sind die Ablation benigner Tumore, Skinresurfacing, Blepharoplastiken, Akne inversa und blutarmes Schneiden. Der $\mathrm{CO}_{2}$-Laser Laser hat beim Skinresurfacing eine stärkere Wirkung als der Erbium:YAGLaser, da durch die Hitzeeinwirkung ein Kollagen-Shrinking-Effekt entsteht, der beim Erbium:YAG-Laser fehlt. Für die Absaugung des Rauches gilt das oben Gesagte. Auch durch die größere Wärmeentwicklung dieses Lasertyps können nicht sicher alle eventuell vorhandenen Viruspartikel inaktiviert werden.

\section{Nd:YAG-Laser (1064 nm)}

Das Licht dieses Lasers wird zwar noch gut von Wasser absorbiert, aber um einige Größenordnungen schwächer als das der beiden anderen Geräte. Dies führt dazu, dass die Energie tief ins Gewebe eindringen und dort ihre Wirkung entfalten kann. Damit erreicht man beispielsweise tiefliegende Gefäße, venöse Malformationen und große Angiome. Eine Blutstillung findet nicht statt. Indikationen in der Dermatologie sind gegeben bei Gefäßbehandlungen, Akne inversa, Epilation und anderen. Da man im Allgemeinen keine direkte Hautreaktion sieht, ist Vorsicht bei der Anwendung geboten, da beim Stacking, also bei der repetitiven Applikation von Impulsen auf die gleiche Stelle, eine Akkumulation der Hitze in der Tiefe entstehen kann und sich leicht Koagulationsnekrosen bilden. Obligat ist eine Oberflächenkühlung, beispielsweise mit Kaltluft oder Cryogenspray.

\section{Nicht zu vergessen: Histologie}

Generell empfiehlt sich vor Anwendung eines abtragenden oder koagulierenden Lasers aus forensischen Gründen unbedingt eine histologische Sicherung der Diagnose. Nach der Laseranwendung kann meist keine verwertbare Histologie gewonnen werden.

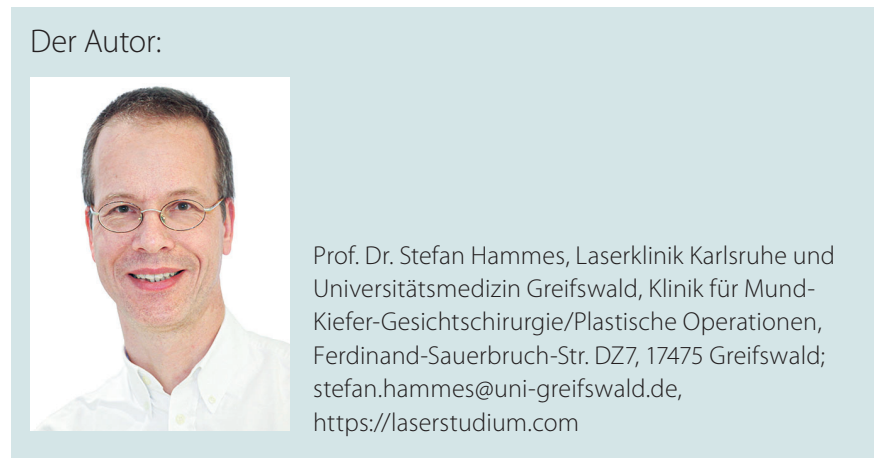




\section{Aus der Praxis}

\section{Arztberuf im Wandel - welche Herausforderungen erwarten uns?}

Wie sieht die Zukunft im Arztberuf aus - vor allem im ambulanten Bereich? Darüber sprach Prof. Dr. Peter Rohmeiss beim Operation Karriere-Kongress in Heidelberg. Sein Rat: Bei aller Planung auch für Zufälle und spontane Entscheidungen offen zu bleiben.

«Uns steht ein gewaltiger Wandel bevor», erklärte Rohmeiss dem Publikum im Hörsaal der SRH Hochschule Heidelberg. Unter anderem seien die geplanten Krankenhausschließungen und der Hausarzt- und Fachärztemangel Themen, die den Arztberuf der Zukunft prägen werden. Doch ein Grund für Schwarzmalerei sei das nicht - Pessimismus habe es auch schon vor 20 Jahren gegeben. Man könne für die Zukunft daher einiges aus der Vergangenheit lernen. Rohmeiss begann mit einem Beispiel aus seinem Fachbereich, der Nephrologie: Während die Nierenheilkunde früher ein rein klinisches Fach gewesen sei, habe sie dann in Form von Dialysepraxen den Weg in den ambulanten Bereich gefunden. Nun kehren die niedergelassenen Fachärzte in die Kliniken zurück und übernehmen dort intensivmedizinische Leistungen, die sich die Kliniken nicht mehr leisten können. In anderen Bereichen, wie der Radiologie, gebe es eine ähnliche Entwicklung. In den Niederlanden sei es beispielsweise schon heute üblich, dass Zusammenschlüsse von niedergelassenen Fachärzten Kliniken ihre Leistungen zur Verfügung stellen.

\section{Klassische Niederlassung - ein Auslaufmodell?}

Rohmeiss wagte auch einen Blick in die Zukunft: Eine Konzentration im Bereich der Primärversorgung auf Krankenhäuser und Polikliniken werde es auch künftig nicht geben - es werde auch weiterhin dezentrale Strukturen und eine wohnortnahe Versorgung geben, prognostizierte er. Allerdings sei der klassische niedergelassene Arzt ein Auslaufmodell; künftig werde es keine Einzelkämpfer mehr geben. Schon jetzt gebe es viele verschiedene Kooperationsmöglichkeiten - von der Anstellung in einer Praxis bis zur Gemeinschaftspraxis.

Rohmeiss selbst ist Geschäftsführer der ze:roPRAXEN GbR - eines Praxenverbundes in der Rhein-Neckar-Region. Hier haben sich niedergelassene Ärzte verschiedener Fachgebiete zu einer größeren Struktur verbunden, die viele verschiedene Arbeitsmodelle ermögliche, erklärte er. Es gebe Teilzeitmodelle, Vollzeitmodelle, Praxisgründungen mit Eigenkapital oder als Juniorpartnerschaft oder auch Ärzte, die ein Leben lang angestellt arbeiten.

Zwei neue Player auf dem medizinischen Spielfeld

Die Zukunft werde vor allem von Teamwork und flacheren Hierarchien geprägt, prophezeite Rohmeiss. Im ambulanten Bereich

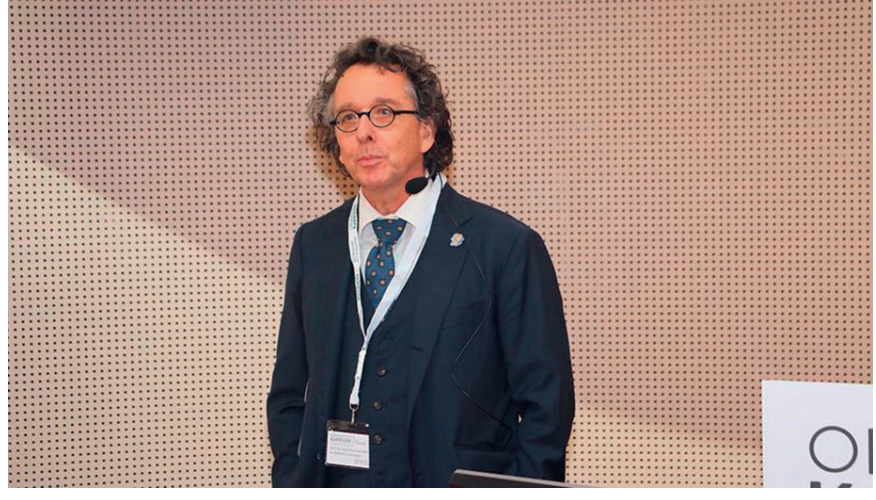

Wie sieht die Zukunft für Mediziner im ambulanten Bereich aus? Beim Operation Karriere-Kongress in Heidelberg wagte Prof. Dr. Peter Rohmeiss einen Blick in die Zukunft. @Hanke

gebe es das schon, aber auch in den Kliniken seien die aktuellen hierarchischen Strukturen nicht zukunftsfähig. Vor allem zwei neue Entwicklungen werden die Zusammenarbeit dabei drastisch verändern:

Akademisierung der Pflegeberufe: Durch neue akademische Berufsbilder wie z.B. Physician Assistans (PAs), aber auch Bachelor, Master, Doktoren und sogar Professoren im Pflegebereich können ärztliche Leistungen künftig stärker delegiert werden.

Künstliche Intelligenz als dritter Player: Außerdem werde es in wenigen Jahren immer mehr und immer bessere digitale Systeme geben, die Diagnosen und andere ärztliche Aufgaben übernehmen können. Schon jetzt gebe es gute Diagnoseprogramme: «ln drei Jahren wird es die ersten Google- und Amazon-Ärzte geben», prophezeite Rohmeiss.

«Patientendaten gehören nur in die Hände von Ärzten und Patienten und sollten nicht an Dritte weitergegeben werden - auch nicht über praktische Apps», forderte Rohmeiss in diesem Zusammenhang. Er beendete seinen Vortrag mit einem hoffnungsvollen Wunsch an die kommende Ärztegeneration: «Seien Sie offen - Ihnen steht eine faszinierende Zukunft bevor.»

Quelle: Operation Karriere-Kongress Heidelberg, 7.12.2019, Vortrag: «Der Arztberuf im Wandel - Perspektiven und Herausforderungen im ambulanten Bereich», Prof. Dr. med. Peter Rohmeiss, Geschäftsführer der ze:roPRAXEN, Schwetzingen. 